\title{
Short term results of anterior cruciate ligament reconstruction using four strand hamstring tendon autograft with endobutton fixation.
}

\author{
1.Moh'd Shafi Bhat. 2.Moh'd Yaseen Rather. 3.Irfan Ahmad Latoo. \\ 4. Azad Ahmad Shah. 5.Iftikhar Hussain Wani. 6. Munir Farooq. \\ 1,2 \& 4: Postgraduate scholars orthopaedics. $3 \& 5$ : Senior residents orthopaedics. \\ 6: Professor and Head of Unit 2. Hospital for Bone and Joint Surgery Barzulla. \\ Postgraduate Department of Orthopaedics; Govt Medical College Srinagar. Kashmir.
}

\begin{abstract}
Objective: To evaluate the results of anterior cruciate ligament (ACL) reconstruction using four strand hamstring tendon autograft.

Settings: Hospital for Bone and Joint Surgery Srinagar Kashmir.

Material and methods: Thirty patients of anterior cruciate ligament tears underwent arthroscopic ACL reconstruction using four strand hamstring tendon autograft between October 2010 to November 2012.

Results: The mean age in our series was 31.8 years with a range from 19-50 years. The male to female ratio was 5:1. Sports injury accounted for $46.7 \%$ of the cases. $46.7 \%$ of the cases had an associated meniscal injury. The Lysholm score improved from a mean pre-op value of 52.7 (range 22-81) to mean value of 89.7 (range 62-100) at the final follow-up at a minimum of 2 years. The single leg hop measurements improved from a mean pre-op value of $52.37 \%$ to mean value of $88.4 \%$ at the final follow-up. $93.3 \%$ of the patients had either grade 0 or grade 1 Lachman grading at the final follow-up.

Conclusion : Arthroscopic ACL reconstruction eliminated symptomatic knee instability associated with a torn ACL. Significant improvements in the functional scores were noted.

Key Words : Anterior Cruciate Ligament; ACL reconstruction; Endobutton fixation ; Hamstring tendon autograft; Semitendinosus autograft; Transtibial technique .
\end{abstract}

\section{Introduction}

The anterior cruciate ligament (ACL) is the most frequently completely disrupted ligament of the knee.The incidence of ACL tears has increased in the general population with the rise of participation in sports. ${ }^{1}$ The development of symptomatic knee instability after ACL injury ranges from $16 \%$ to almost $100 \%{ }^{2}$ Without treatment, a complete ACL injury can result in progressively increasing symptomatic knee instability, which inflicts recurrent intra-articular damage and eventually causes osteoarthritis. The fundamental rationale for the surgical reconstruction of the torn ACL is to stabilize the knee and prevent further meniscal tears and associated joint damage. ${ }^{3} \mathrm{Graft}$ choices for ACL reconstruction include autografts and allografts. Prosthetic materials have also been used. For many years, the BTB ( bone-tendon-bone) autograft has been advocated as the gold standard but the issues relating to donor site problems have led to the increased use of STG/ST autografts. The quadruple hamstring tendon autograft has higher graft strength, stiffness and cross-section area compared to the BTB graft, and, additionally, the extensor mechanism is preserved. ${ }^{4}$

\section{Material and Methods}

The study was conducted in Hospital for Bone and Joint Surgery Barzulla Srinagar, an associated hospital of Govt. Medical College Srinagar. The prospective study consists of a total of 30 cases who underwent arthroscopic ACL reconstruction between Oct 2010 to Nov 2012 using four strand semitendinosus autograft. All the cases were operated by the senior author ( Munir Farooq ). The operating surgeon performed no other type of reconstruction during the study interval. Trans-tibial technique was employed in all the cases. Graft fixation on the femoral side was done using an endobutton CLS in all the 30 cases while tibial side was fixed using the suture disc .Patients in the age-group of 18-50 years with symptomatic ACL tears were included in the study. Patients with bilateral knee injuries, revision ACL reconstruction and patients with associated fractures around the knee were excluded from the study.

The pre-operative assessment included detailed history and physical examination, radiographs and MRI of the involved knee. Lachman test ,Anterior drawer test and Mc Intosh Pivot shift test were used for testing ACL . The results were graded as 0 (negative), $1+, 2+$ and $3+$ positive. Other tests performed routinely included the Varus and Valgus stress tests ,Mc Murray's test, Posterior drawer test and the Reverse Pivot shift test. Radiographs included the standing AP view of both the knees and a Lateral and Notch view of the affected 
knee. Notch view was done to determine the notch width.MRI of the involved knee was done in all the cases ( Fig 1). Lysholm scoring, Tegner activity level and single leg hop were done and documented. Circumferential measurements of the calf and thigh were taken at defined landmarks.

\section{Operative Technique}

Antibiotics were given $1 \mathrm{hr}$ before the surgery. The patient was placed supine on the operating table with the knee joint positioned slightly past the distal breakpoint of the table. The opposite limb was well padded. Examination of the patient under anaesthesia was done and the correct limb marked . Pneumatic tourniquet was applied to the operative leg after exsanguination. Graft harvesting was performed through an oblique incision at the pes insertion. The graft was harvested using a tendon stripper. Graft preparation was done with the removal of the residual muscle from the harvested tendon. The graft was divided into two equal parts. The graft ends were whip-stitched at each end for 30 to $40 \mathrm{~mm}$ with Ethibond suture (Fig. 2). The two strands were folded in the middle to form a quadruple graft. The construct was put on a graft tensioner till we proceeded with tunnel preparation.

Standard anterolateral and anteromedial arthroscopy portals were used. Remnants of torn ACL were debrided ( Fig. 3). Femoral tunnel was prepared through the tibial tunnel. It required visualization of the overthe-top position . A placement guide that keys off the over-the -top position was used ( Fig. 4 ). The posterior border of anterior horn of the lateral meniscus was used as a landmark for intra-articular tibial tunnel placement ( Fig. 5). Tibial drill guide was used for tibial tunnel preparation.The femoral tunnel was reamed $5 \mathrm{~mm}$ deeper than the graft insertion length to allow for the 'turning radius' of the endobutton. The tibial tunnel entry was made as close to the tibial attachment of superficial part of MCL as possible to make the tunnel more horizontal. The graft was transferred from the masterboard and appropriate length CLS with endobutton was attached to the graft. The graft was marked with a sterile marking pen. A passing pin was advanced through the tunnels and graft construct pulled proximally. Endobutton was flipped on the anterolateral femoral cortex . The knee was taken through a full range of motion . The tibial fixation was done using a suture disc( Fig. 6) . ${ }^{5}$

Patients were followed up for a minimum of 24 months. Patients followed a post ACL reconstruction accelerated rehabilitation protocol. ${ }^{6}$ Final assessment was done on the basis of stability tests i.e, Lachman test, Anterior drawer test and McIntosh Pivot shift tests. Lysholm scoring, Tegner activity level and Single leg hop measurements were done in addition to the subjective assessment of the patients . Tunnel position was assessed on the post-op radiographs ( Fig. 7 a,b ). Tunnel position was determined using the Harner's Quadrant Method .

\section{Results}

The mean age in our series was 31.8 years with a range from 19-50 years. The male to female ratio was $5: 1$ with a total of 25 males and 5 females. Right knee was involved in 16 cases $(53.3 \%)$ and left knee in 14 cases (46.7\%). Sports related injuries accounted for majority of the cases (46.7\%) . Vehicular accidents accounted for $23.3 \%$ of the cases.(Table 1).The median time from injury to surgery was 15 months with a range from 2 months to 8 years. The median hospital stay in our series was 4 days with a range of 3-5 days. Regional anaesthesia was used in most of our cases (93.3\%). The mean time taken for surgery was 94.5 minutes with values ranging from 70 to 130 minutes. 14 patients $(46.67 \%$ ) had associated meniscal injuries.(Table 2 ).

The Lysholm score improved from a pre-operative mean of 52.7 ( range 22-81) to a mean value of 89.7 ( range 62-100) at the final follow up. The mean Lysholm score in patients with associated meniscal injury was 88.1 at the final follow-up compared to the mean score of 91.2 in the group without associated meniscal injury. The Single leg hop measurements improved from a mean pre-op value of $52.37 \%$ ( range $0-71$ ) to mean value of $88.4 \%$ ( range $68-108$ ) at the final follow-up.

\section{Discussion}

Surgical reconstruction of the ACL has become the standard in the treatment of ACL injuries. The primary goal of ACL reconstruction is to restore the stability of the knee .Successful clinical outcomes following anterior cruciate ligament reconstruction with a hamstring graft has been reported by many authors ${ }^{8,9}$ It is difficult to compare the results of existing studies because of variations in the reported surgical techniques. ${ }^{10,11}$ Nebelung et al reviewed the results of twenty-nine anterior cruciate reconstructions with a doubled autogenous semitendinosus tendon and a femoral endobutton. They graded $66 \%$ of the results as normal or nearly normal. ${ }^{12}$ In the present series of thirty patients $93.3 \%$ of the patients had either Grade 0 or Grade 1 Lachman values at the final follow-up.The comparison of Lachman test values at the final follow-up is shown in Table 3.

Lysholm score in the present series improved from a mean pre-op value of 52.7 to mean value of 89.7 at the final follow-up.In S Plaweski et al 2009 series with a mean follow-up time of 51 months the Lysholm score improved from a mean pre-op value of 72.1 ( range 50-86) to mean value of 94.1 ( range 71-99) at the final follow-up. ${ }^{13}$ In Ejerhed L et al series the median Lysholm score pre-op was 68 ( range 21-100) and 90 
(range 51-100) at the final follow-up at 2 years. ${ }^{14}$ In Eriksson et al series 2001 with a median follow-up time of 31 months the Lysholm score improved from a mean pre-op value of 71 ( range 29-90) to mean value of 86 ( range 45-100) at the final follow-up. ${ }^{15}$

Sports trauma accounted for majority of the cases in most of the series reported in the literature. In the present series sports related activities was the commonest mode of injury comprising $46.7 \%$ of the patients. Road traffic accidents accounted for $23.3 \%$ of the patients. Meniscal injuries associated with ACL tears were found in $14(46.67 \%)$ out of 30 cases in the present series. H Arsi et al 2006 reported a series of 39 patients out of which 12 ( $30.7 \%$ ) had meniscal tears. The time since injury in their study was 16 months. ${ }^{16}$ Ejerhed L et al 2003 reported a series of 34 patients , 24 (70.6\%) of which had meniscal injuries. ${ }^{14}$

Range of motion was compared with the contralateral knee. At the final follow-up 17 patients had no motion deficit. 6 patients had isolated flexion deficit of 10 degrees or less. 4 patients had isolated extension deficit of 5 degrees or less. 3 patients had both flexion and extension deficits at the final follow-up. In $\mathrm{S}$ Plaweski et al series of 105 patients 4 patients had 5 degrees of extension deficit. 7 patients had a mean flexion deficit of 7 degrees. ${ }^{13}$ In Ejerhed L et al 2003 series loss of extension of 5 degrees or more compared to the healthy contralateral side was registered in 9 of 34 patients and flexion deficit of 5 degrees was found in 24 of 34 patients. $^{14}$

Pinczewski et al 2008 in a study of ACL reconstruction concluded that if the osseous tunnels are correctly placed, single bundle ACL reconstruction adequately controls both anteroposterior and rotational instability. ${ }^{17}$ Tunnel localization on the post-op x-rays was determined using the Harner quadrant method. Tibial tunnels were in quadrant 2 in all the 30 patients in the saggital plane. In the coronal plane the tibial tunnel centre was located in quadrant 2 in 22 and in quadrant 3 in 8 patients. Femoral tunnel was localized in quadrant 4 in 28 and in quadrant 3 in 8 of the 30 cases. In a study by Harner CD et al all the cases had tibial tunnel in quadrant 2. 22 of 26 cases had femoral tunnel in quadrant 4 and 4 in quadrant 3 . The study checked only the saggital tunnel placement. ${ }^{7}$

Complications included superficial wound infection which developed in two ( $6.67 \%)$ out of 30 patients at the tibial fixation site. Both the cases were managed with antiseptic dressings and oral antibiotics for 1 week. 12 ( $40 \%$ ) patients complained of numbness over the anterior aspect of leg. In a study by Spicer et al areas of sensory change over the front of the knee were identifiable in $50 \%$ of the patients and of these $86 \%$ demonstrated sensory changes in the distribution of the infragenicular branch of the saphenous nerve. ${ }^{18}$

\section{Conclusion}

Arthroscopic ACL reconstruction using quadrupled semitendinosus autograft eliminated symptomatic knee instability associated with a torn anterior cruciate ligament. Significant improvements in functional scores were noted. Accelerated rehabilitation was possible in view of strong suspensory fixation using the endobutton. There were no major complications of either the surgical procedure adopted or the fixation method used.

\section{Bibliography}

[1]. Apostolopoulos A, Nakos A, Nikolopaulos D, Theophanopaulos F, Liarokapis S et al. ACL reconstruction with hamstring tendon autografts. E.E.X.O.T.2009. Volume 60. Number 1, 48-52.

[2]. Frank CB , Jackson DW . The science of reconstruction of the anterior cruciate ligament . J Bone Joint Surg Am 1997; 79: 11561576 .

[3]. Daniel DM , Stone ML, Dobson BE, et al. Fate of the ACL injured patient: a prospective outcome study. Am J Sports Med 1994;22:632-644.

[4]. Brown $\mathrm{CH}$, Steiner ME, Carson EW. The use of hamstring tendons for anterior cruciate ligament reconstruction: techniques and results. Clin Sports Med 1993;12:723-756.

[5]. Alberto Gobbi , Ramces Francisco. Hamstring anterior cruciate ligament reconstruction with a quadrupled or tripled semitendinosus tendon graft. Prodromos:The anterior cruciate ligament: Reconstruction and basic science ( $1^{\text {st }}$ edition .2008 Saunders).

[6]. Shelbourne KD, Nitz P . Accelerated rehabilitation after ACL reconstruction. Am J Sports Med 1990; Vol 18 No 3 :292-299.

[7]. Harner CD, Marks PH, Fu FH,Irrgang JJ, Silby MB , Mengato R. Anterior cruciate ligament reconstruction :Endoscopic versus two-incision technique. Arthroscopy 1994: 10(5) : 502-512.

[8]. Aglietti P, Buzzi R, Zaccherotti G, De Biase P : Patellar tendon versus doubled semitendinosus and gracilis tendons for ant erior cruciate ligament reconstruction.Am J Sports Med 1994; 22: 211-217.

[9]. Aglietti P, Buzzi R, Menchetti P M , Giron F . Arthroscopically assisted semitendinosus and gracilis tendon graft in reconstruction for acute anterior cruciate ligament injuries in athletes. Am J Sports Med 1996; 24 : 726-731.

[10]. Maeda A, Shino K, Horibe S et al . Anterior cruciate ligament reconstruction with multistranded autogenous Semitendinosus tendon. Am J Sports Med 1996; 24 : 504-509.

[11]. Zysk SP, Kruger A, Baur A, Veihelmann A, Refior HJ . Tripled semitendinosus anterior cruciate ligament reconstruction with Endobutton fixation. Acta Orthop Scand 2000; 71 (4) : 381-386

[12]. Nebelung W, Becker R, Merkel M, Ropke M . Bone tunnel enlargement after anterior cruciate ligament reconstruction with semitendinosus tendon using endobutton fixation on the femoral side.Arthroscopy.1998;14:810-5.

[13]. Plaweski S , Rossi J , Meeloz P . ACL reconstruction : Assessment of the hamstring autograft femoral fixation using the Endobutton CL. Orthopaedics and Traumatology : Surgery and Research (2009). 95: 606-613.

[14]. Ejerhed Lars, Kartus Juri, Sernert N, Kohler K, Karlsson J . Patellar tendon or semitendinosus tendon autografts for anterior cruciate ligament reconstruction? Am J Sports Med 2003; Vol 31. No 1:19-25. 
[15]. Eriksson K, Anderberg P, Hamberg P, Lofgren AC, Bredenberg M, Westman I , Wredmark T et . A comparison of quadruple semitendinosus and patellar tendon grafts in reconstruction of the anterior cruciate ligament. J Bone Joint Surg Br 2001 Apr;83 (3):348-354

[16]. Harilainen Arsi , Linko E, Sandelin J. Randomized prospective study of ACL reconstruction with interference screw fixation in patellar tendon autografts versus femoral metal plate suspension and tibial post fixation in hamstring tendon autografts. Knee Surg Sports Traumatol Arthrosc 2006; 14(6) : 517-528.

[17]. Pinczewski LA, Salmon LJ, Jackson WF, von Bormann RB, Haslam PG, Tashiro S. Radiological landmarks for placement of the tunnels in single-bundle reconstruction of the ACL. Journal Bone Joint Surg Br 2008; 90-B : 172-179.

[18]. Spicer DDM , Blagg SE, Unwin AJ , Allum RL. Anterior knee symptoms after four-strand hamstring tendon anterior cruciate ligament reconstruction. Knee Surg Sports Traumatol Arthrosc (2000) 8: 286-289

Figures and Tables

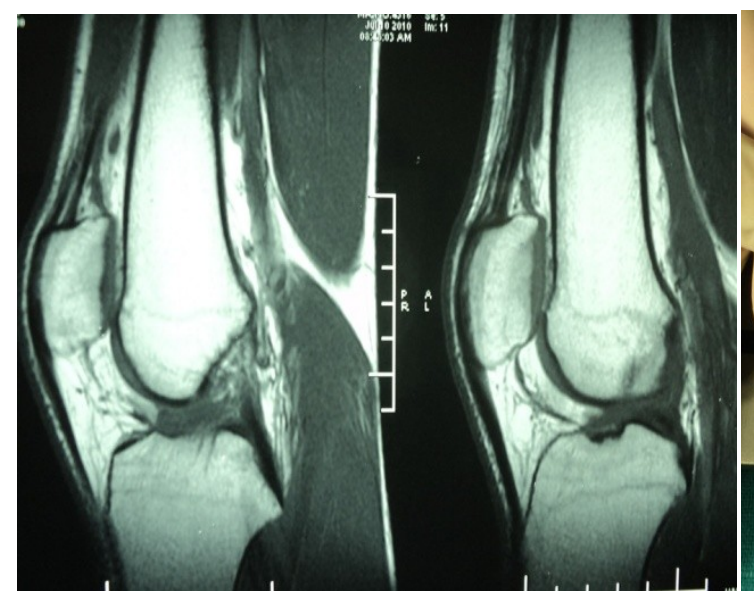

Figure 1. Saggital MR images showing ACL tear.

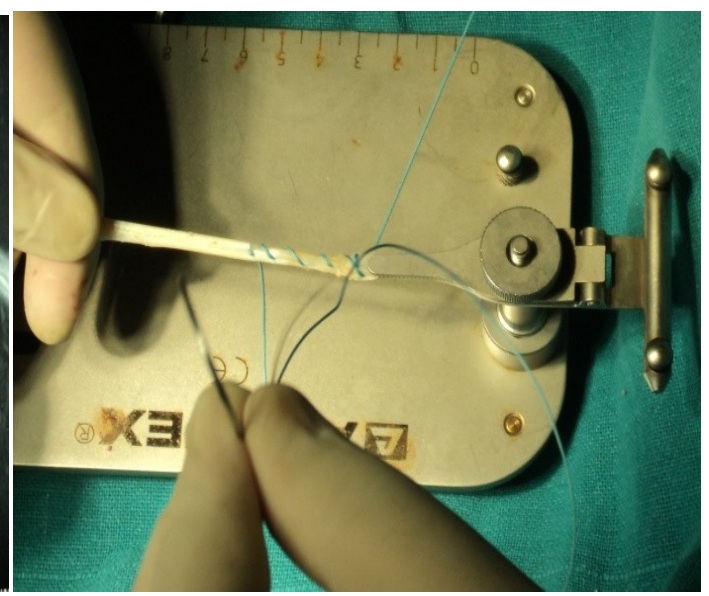

Figure 2. Graft preparation on masterboard.

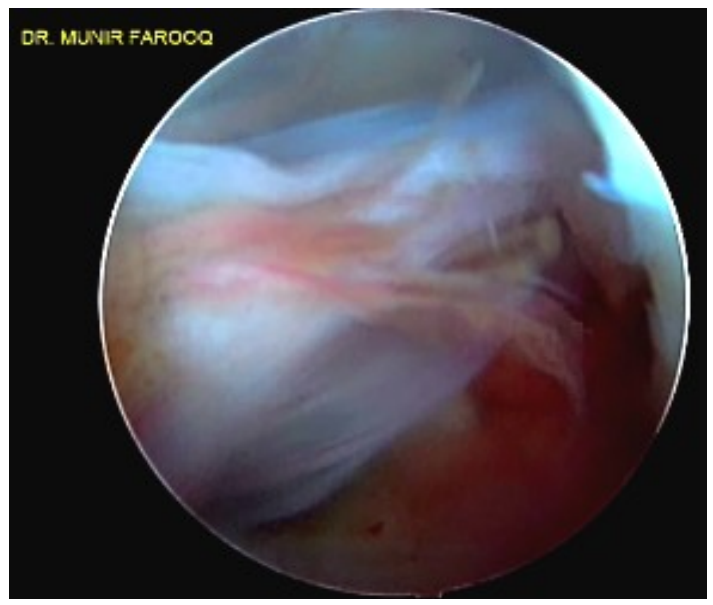

Figure 3 . Arthroscopic picture of $A C L$ remnant. 

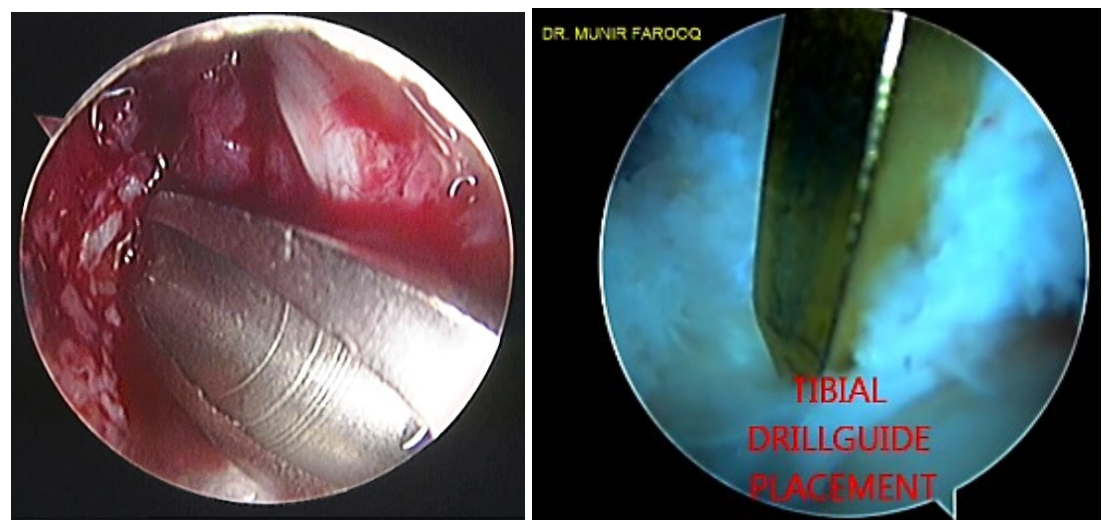

Figure 4 . Femoral aimer placement.

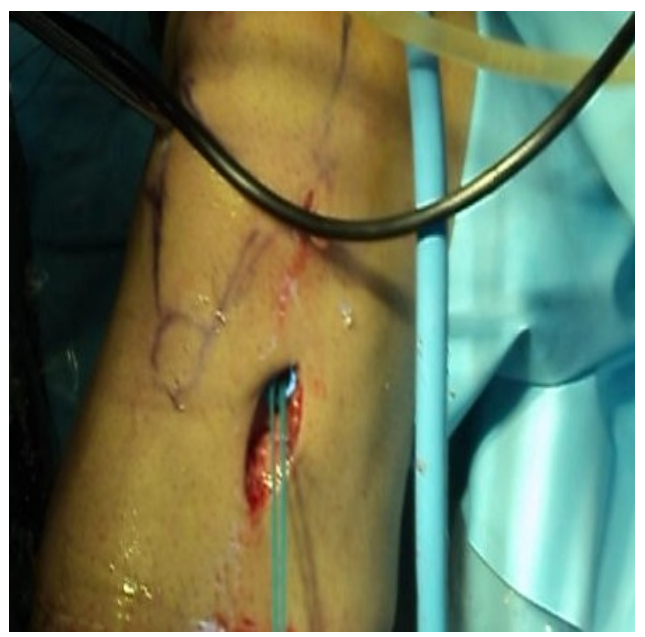

Figure 6.Tibial fixation using suture disc.

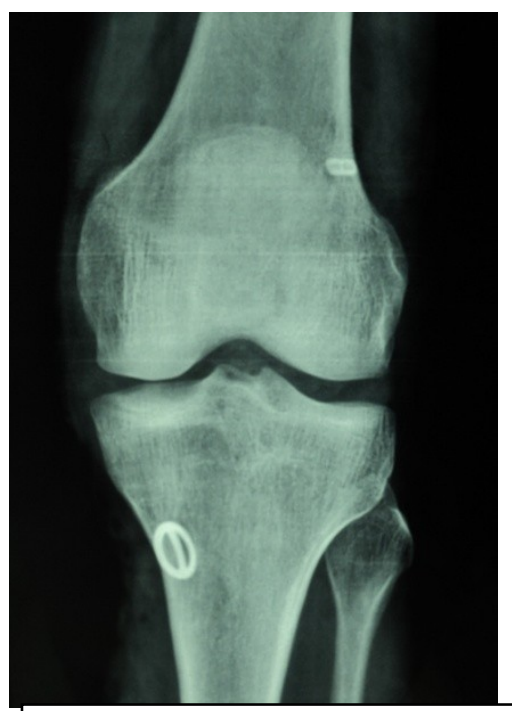

Figure 7a. X-ray Knee ( AP view ) post-operative.
Figure 5. Tibial drillguide placement (intra-articular).

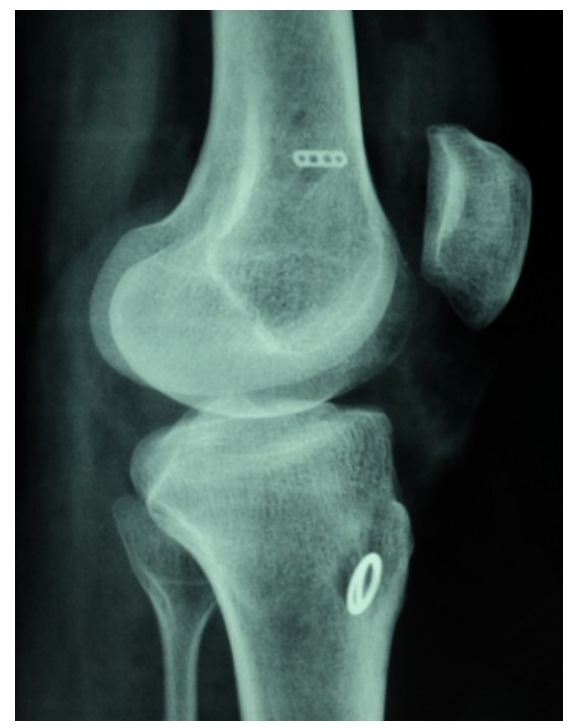

Figure $7 b$. X-ray Knee (lateral view) post-operative. 
TABLE 1

Table showing the mode of ACL injury.

\begin{tabular}{|l|l|l|l|}
\hline $\begin{array}{c}\text { S. } \\
\text { No. }\end{array}$ & Mode of Injury & No. of cases & Percentage \\
\hline 1 & Sports related injuries & 14 & 46.7 \\
\hline 2 & Road traffic accidents height/fall on uneven & 7 & 23.3 \\
\hline 3 & $\begin{array}{l}\text { Fall from } \\
\text { ground }\end{array}$ & 4 & 16.7 \\
\hline 4 & Others & 30 & 13.3 \\
\hline & Total & 100 \\
\hline
\end{tabular}

Table 2

Table showing the pattern of meniscal injuries .

\begin{tabular}{|c|c|c|c|}
\hline $\begin{array}{l}\text { S. } \\
\text { No. }\end{array}$ & Meniscus involved & $\begin{array}{l}\text { No. of } \\
\text { patients }\end{array}$ & Percentage \\
\hline 1 & No meniscal injury & 16 & 53.3 \\
\hline 2 & $\begin{array}{l}\text { Medial meniscal } \\
\text { injuries }\end{array}$ & 11 & 36.7 \\
\hline 3 & $\begin{array}{ll}\text { Lateral meniscal } \\
\text { injuries }\end{array}$ & 2 & 6.7 \\
\hline \multirow[t]{2}{*}{4} & $\begin{array}{l}\text { Both menisci } \\
\text { involved }\end{array}$ & 1 & 3.3 \\
\hline & Total & 30 & 100 \\
\hline
\end{tabular}

Table 3

Table showing the comparison of pre-op and final follow-up Manual Lachman grading.

\begin{tabular}{|l|l|l|l|l|l|}
\hline \multirow{2}{*}{ S. No. } & Manual Lachman test & Pre-operative & \multicolumn{2}{l|}{ At final follow up } \\
\cline { 2 - 6 } & & No of patients & Percentage & No of patients & Percentage \\
\hline 1. & 0 & 0 & 0 & 21 & 70.0 \\
\hline 2. & 1 & 0 & 0 & 7 & 23.3 \\
\hline 3. & 2 & 11 & 36.7 & 2 & 6.7 \\
\hline 4. & 3 & 19 & 63.3 & 0 & 0.0 \\
\hline & Total & 30 & 100 & 30 & 100 \\
\hline
\end{tabular}

